\title{
Evaluating the clinical utility and sensitivity of SARS-CoV-2 antigen testing in relation to RT-PCR Ct values
}

\author{
Lukas Lanser $^{1} \cdot$ Rosa Bellmann-Weiler $^{1} \cdot$ Karla-Wanda Öttl| $^{1} \cdot$ Lukas Huber $^{1} \cdot$ Andrea Griesmacher $^{2} \cdot$ Igor Theurl $^{1}$. \\ Günter Weiss ${ }^{1}[0$
}

Received: 4 October 2020 / Accepted: 21 October 2020 / Published online: 13 November 2020

○) Springer-Verlag GmbH Germany, part of Springer Nature 2020

To the Editor,

Diagnosis of severe acute respiratory syndrome coronavirus 2 (SARS-CoV-2) in patients with suspected coronavirus disease 2019 (COVID-19) is most widely performed with real time polymerase chain reaction (RT-PCR) considered as gold standard [1]. RT-PCR is a highly sensitive diagnostic method for detecting viral ribonucleic acid (RNA) with the disadvantages of logistics for transport of samples to specific laboratories and the relative long duration of the diagnostic method. Positive SARS-CoV-2 RT-PCR also do not allow definitive conclusions whether the subject is still contagious or not. This can be partly attained by establishing the cycle threshold $(\mathrm{Ct})$ value depicting the particular amount of viral RNA in the sample and thus allowing a conclusion on the viral load and infectivity [2]. However, because of the diagnostic effort and duration of the test, faster and less laborious tests attract interest which may help to rapidly identify and contain infected individuals. Therefore, point-of-care-testing with antigen tests, providing results in a couple of minutes, is currently evaluated for routine clinical use.

We compared the SARS-CoV-2 antigen detection in nasopharyngeal swab samples by the Panbio ${ }^{\text {TM }}$ COVID-19 Ag Rapid test (Abbott, Chicago, Illionis) with the simultaneous routinely conducted RT-PCR analysis of SARS-CoV-2 orf1 RNA detection with the cobas ${ }^{\circledR}$ analyzer (Roche Diagnostics GmbH, Mannheim, Germany). The nasopharyngeal swab samples were collected from 53 patients with PCRconfirmed SARS-CoV-2 infection during their hospital stay in different stages of the disease. Panbio ${ }^{\text {TM }}$ COVID-19 Ag

Günter Weiss

guenter.weiss@i-med.ac.at

1 Department of Internal Medicine II, Innsbruck Medical University, Anichstraße 35, 6020 Innsbruck, Austria

2 Central Institute for Medical and Chemical Laboratory Diagnosis, Innsbruck University Hospital, Innsbruck, Austria
Rapid test was performed right after nasopharyngeal swab sampling while RT-PCR was routinely performed in our central laboratory facility. RT-PCR was negative in two patients suggesting an already subsided infection; consistent with it the Panbio ${ }^{\text {TM }}$ COVID-19 Ag Rapid test was also negative. Among 51 RT-PCR SARS-CoV-2 positive patients, the Panbio $^{\text {TM }}$ COVID-19 Ag Rapid test was positive in 31 subjects depicting a poor sensitivity of $60.8 \%$ (95\% CI $46.1-74.2 \%$ ), compared to $93.3 \%$ in the manufacturer's information. In the 14 patients with a $\mathrm{Ct}$-value $\leq 25$, being indicative for higher viral loads, the sensitivity for the Panbio ${ }^{\mathrm{TM}}$ COVID-19 Ag Rapid test was at a level of $85.7 \%$ (95\% CI 57.2-98.2\%, Table 1). Panbio ${ }^{\mathrm{TM}}$ COVID-19 Ag Rapid test was positive in $36.4 \%$ (95\% CI $17.2-59.3 \%)$ of the patients with a Ctvalue $>30$ (Table 1). Of note, when we included subjects with a $\mathrm{Ct}$ value $\leq 30$, which is considered to be a threshold for infectivity and currently recommended by the German Robert Koch Institute as an important cutoff to identify SARS-CoV2 contagious subjects, we found that the $\mathrm{Ag}$ rapid test correctly identified $79.3 \%$ of individuals. However, looking on the other site of the coin we found that subjects with a Ct-value $>30$ were antigen positive in $36.4 \%$ of cases. The test is still positive in a considerable number of patients which are considered as being non-infectious according to the German Robert Koch Institute. Changing the cut-off value to $\mathrm{Ct}>33$ reduced the positive results of the Panbio $^{\text {TM }}$ COVID-19 Ag Rapid test to $16.7 \%$ (Table 1) whereas no positive Panbio ${ }^{\text {TM }}$ COVID-19 Ag Rapid test result was achieved in subjects with RT-PCR Ct-values $\geq 35(n=4)$.

These results indicate a poor performance of the Panbio $^{\text {TM }}$ COVID-19 Ag Rapid test detecting SARS-CoV-2 antigen in patients with low virus load but a good performance in patients with high virus load. Viral load of SARS$\mathrm{CoV}-2$ was reported to be the highest around the time of symptom onset, and most probably become undetectable within approximately 2 weeks. Additionally, patients with more severe symptoms also seems to have a higher viral 
Table 1 Antigen test results stratified by different $\mathrm{Ct}$ value cut-offs

\begin{tabular}{lcrc}
\hline & $\begin{array}{l}\text { Antigen test negative } \\
n(\% \text { within Ct value })\end{array}$ & $\begin{array}{l}\text { Antigen test positive } \\
n(\% \text { within Ct value })\end{array}$ & $\begin{array}{l}\text { Total } \\
n(\% \text { within total) }\end{array}$ \\
\hline Ct value $>25$ & $18(48.6 \%)$ & $19[51.4 \%(95 \%$ CI 34.4-68.1)] & $37(72.5 \%)$ \\
Ct value $\leq 25$ & $2(14.3 \%)$ & $12[85.7 \%(95 \%$ CI 57.2-98.2)] & $14(27.5 \%)$ \\
Ct value $>30$ & $14(63.6 \%)$ & $8[36.4 \%(95 \%$ CI 17.2-59.3)] & $22(43.1 \%)$ \\
Ct value $\leq 30$ & $6(20.7 \%)$ & $23[79.3 \%(95 \%$ CI 60.3-92.0)] & $29(56.9 \%)$ \\
Ct value $>33$ & $5(83.3 \%)$ & $1[16.7 \%(95 \%$ CI $0.42-64.1)]$ & $6(11.8 \%)$ \\
Ct value $\leq 33$ & $15(33.3 \%)$ & $30[66.7 \%(95 \%$ CI 51.1-80.0)] & $45(88.2 \%)$ \\
Total & $20(39.2 \%)$ & $31[60.8 \%(95 \%$ CI 46.1-74.2)] & $51(100 \%)$ \\
\hline
\end{tabular}

loads [3, 4]. Therefore, antigen tests, like the one investigated herein, might be suitable and effective for rapidly identifying infectious subjects with symptoms compatible to a COVID-19 infection in a primary care setting given its sensitivity of around $80 \%$. This is of relevance specifically in epidemic situations with numerous other circulating virus such as influenza but also for rapidly identifying SARS-CoV-2 positive patients for immediate initiation of containment and contact tracing strategies for better controlling the spread of the infection mainly during the cold season [5]. Moreover, the test may be of specific benefit in subjects with short onset of symptoms as viral loads are highest during the first days of infection and because the test has been shown herein to have an even higher sensitivity in patients with higher viral loads. However, one has to admit that not all subjects with a $\mathrm{Ct}$-value $\leq 30$ have been correctly identified as being SARS-CoV-2 infected. This would warrant the development of additional strategies to minimize the risk of false negative results. Such algorithms could include the performance of a RT-PCR test in subjects with symptoms compatible with COVID-19 but a negative antigen result. The discrepancy between the real sensitivity and that claimed by the manufacturer of SARS-CoV-2 Ag tests was already demonstrated recently [6]. Since these are CE-marked diagnostic devices this question the reliability of the European admission process.

The low number of positive antigen test results in subjects with higher $\mathrm{Ct}$ value $>33$ which are considered to be not contagious (https://www.cdc.gov/coronavirus/2019ncov/hcp/duration-isolation.html) could have implications for follow up screening of initially antigen positive subjects to determine the time point when they are no longer contagious, which is specifically relevant for estimating the duration of quarantine measures. Whether or not this antigen test is suitable for public screening measures mostly of asymptomatic patients to identify undetected subjects with COVID-19 without generating a considerable number of false positive results remains to be shown. Although our results are quite encouraging regarding the use of antigen test as point-of-care diagnostic which may contribute to a better control of the pandemic, we need longitudinal studied which larger patient cohorts to corroborate our results and to develop algorithms or to identify those subjects who are contagious but not detected by that test.

Author contributions Conceptualization and methodology, GW, IT and RBW; Software and formal analysis, LL; Investigation and data curation, CWO, LH and RBW; resources, AG, GW, IT and RBW; Writing-original draft preparation, LL; Writing-review and editing, AG, CWO, GW, IT, LH and RBW; Supervision, GW. All authors have read and agreed to the final version of the manuscript.

Funding There was no funding source for this study.

\section{Compliance with ethical standards}

Conflict of interest The authors declare no conflict of interest.

Ethics approval This study conformed to the principles outlined in the Declaration of Helsinki and was approved by ethics committee of the Innsbruck Medical University (ID of ethical vote: 1167/2020).

Consent to participate As the study was retrospective it was exempt from informed consent.

\section{References}

1. World Health Organisation. Laboratory testing for coronavirus disease (COVID-19) in suspected human cases: WHO/ COVID-19/laboratory/2020.5. https://www.who.int/publicatio ns/i/item/10665-331501

2. Bullard J, Dust K, Funk D, Strong JE, Alexander D, Garnett L, et al. Predicting infectious SARS-CoV-2 from diagnostic samples. Clin Infect Dis. 2020. https://doi.org/10.1093/cid/ciaa638.

3. Walsh KA, Jordan K, Clyne B, Rohde D, Drummond L, Byrne $\mathrm{P}$, et al. SARS-CoV-2 detection, viral load and infectivity over the course of an infection. J Infect. 2020;81:357-71. https://doi. org/10.1016/j.jinf.2020.06.067.

4. Rao SN, Manissero D, Steele VR, Pareja J. A systematic review of the clinical utility of cycle threshold values in the context of COVID-19. Infect Dis Ther. 2020;9:573-86. https://doi. org/10.1007/s40121-020-00324-3.

5. Dzien A, Dzien-Bischinger C, Lechleitner M, Winner $\mathrm{H}$, Weiss G. Will the COVID-19 pandemic slow down in the Northern 
hemisphere by the onset of summer? An epidemiological hypothesis. Infection 2020;48:627-9. https://doi.org/10.1007/ s15010-020-01460-1.

6. Liotti FM, Menchinelli G, Lalle E, Palucci I, Marchetti S, Colavita F, et al. Performance of a novel diagnostic assay for rapid
SARS-CoV-2 antigen detection in nasopharynx samples. Clin Microbiol Infect. 2020. https://doi.org/10.1016/j.cmi.2020.09.030. 